\title{
INFRARED IMAGING OF INTERMEDIATE AGE LMC/SMC CLUSTERS
}

\author{
H. ZINNECKER ${ }^{1}$, F. FERRARO ${ }^{2}$, F. FUSI PECCI ${ }^{3}$, ALVIO RENZINI ${ }^{3}$, \\ R. BUONANNO ${ }^{4}$, C.E. CORSI ${ }^{4}$, D.M. TERNDRUP5 \\ 1. University of Würzburg, Germany \\ 2. ESO Garching, Germany \\ 3. University of Bologna, Italy \\ 4. Astronomical Observatory of Rome, Italy \\ 5. Ohio State University, USA
}

\begin{abstract}
Infrared colour-magnitude diagrams $(K$ vs. $J-K)$ have been obtained for eight intermediate age $(\sim 0.1-1 \mathrm{Gyr})$ Large Magellanic Cloud (LMC) clusters and one Small Magellanic Cloud (SMC) cluster (NGC 419). The data have been obtained from infrared array camera images taken at the CTIO $1.5 \mathrm{~m}$ telescope and reduced using the software package ROMAFOT. We believe that we have observed the onset of the extended red giant branch, i.e. the discontinuous change in the infrared colour and in the number of infrared bright stars per unit optical cluster luminosity. The most luminous stars $(K=10-12.5)$ must be on the asymptotic giant branch; some of them are very red $(J-K>1.5)$ and must be carbon stars.
\end{abstract}

\section{Motivation and Object Selection}

Globular Clusters in the Magellanic Clouds offer a unique tool for testing several predictions of stellar evolution theory and for calibrating models for the evolution of the integrated properties of stellar populations, in particular young galaxies at cosmological redshifts. Some time ago, some of us started an optical CCD study of intermediate age clusters in the LMC, selected for having intermediate B-V colours (between 0.3 and 0.6 ) and/or for being of SWB type IV. These selection criteria stem from the fact that clusters of SWB type III or earlier lack both an extended Asymptotic Giant Branch (AGB) and the Red Giant Branch (RGB); clusters of type V or later show both a well-developed AGB and RGB. The first appearance of the AGB and of the RGB than has to take place within the program clusters. These include (in order of increasing MS-TO age ranging from $\sim 0.1$ to 1 or 2 Gyr): NGC 1831, 1868, 2209, 1987, 2108, 1783, 2162, 2173 (in the LMC) and NGC 419 (in the SMC).

\section{Observations and data reduction}

The infrared imaging of the nine clusters was carried out on three nights in November 1988 at the CTIO $1.5 \mathrm{~m}$ using a $58 \times 62 \mathrm{InSb}$ infrared array camera. The image scale was $0.92 " / \mathrm{pixel}$ and the field of view was about $1^{\prime} \times 1^{\prime}$. The weather conditions were stable and the seeing was around 1.2" The integration time per field was typically 2-3 minutes in each band. Mosaics of $2 \times 2$ frames with some overlapping area were built up to cover the central regions of the clusters (joined together using MIDAS at ESO). The software package ROMAFOT, now optimized for undersampled images, was used to obtain the photometry for our somewhat undersampled images. Seven photometric standard stars from the 1982 list of Elias were observed throughout the three 
nights. Random photometric errors were measured to be within $0.03-0.04$ mag for the standards. Thus the photometric accuracy of our data should be of the order of 5\% for the brighter cluster stars and somewhat less for the fainter ones. Infrared colours should be accurate to $\sim 10 \%$.

\section{Results}

The infrared colour-magnitude diagrams of the nine clusters have been obtained down to a limiting magnitude $K \simeq 16$. To get an indication of the ago of the program clusters we make use of age calibration of the so-called $S$-parameter provided by Elson and Fall (1985), i.e. $\log t=S / 20+7.0$ (somewhat different from the calibration of Chiosi et al. 1988). In a preliminary inspection of the colour-magnitude diagram we have considered two aspects: 1) the number of infrared stars brighter than a certain $K$ magnitude ( say $K=14$ ), and 2) the median infrared colour for stars brighter than a certain $K$ magnitude (say $K=16$ ).

An inspection of the diagrams reveals that the median $J-K$ colour for the youngest clusters in the sample (NGC 1831 and 1868) is rather blue $(J-K<1)$, while for the somewhat older clusters (starting with NGC 1987) the colour becomes progressively redder $(J-K>1)$. In our sample NGC 1987 seems to be the cluster with the critical age for the sudden occurrence of rather red stars. The age of NGC 1987 is $\sim 0.6 \mathrm{Gyr}$ according to the $S$-calibration above, which is in agreement with the age at which standard stellar evolution theory predicts the occurrence of the socalled red giant branch phase transition (Sweigart et al. 1990).

In support of this interpretation we also find that the number of infrared bright stars $(10<K<$ 14) per unit visual cluster luminosity is about a factor of 2 or more in NGC 1987 compared to younger clusters, i.e. clusters younger than NGC 1987 have significantly fewer infrared bright stars than NGC 1987 and the other older clusters. Again, NGC 1987 seems to be the cluster closest to the critical age for a rapid change in the content of LUMINOUS red stars. One caveat is, however, in order. NGC 1987 has the worst field contamination among all the program clusters, and further detailed analysis is necessary to confirm the preliminary conclusion advocated in this report.

Further work is also necessary to compare these results with the previous surveys of luminous red stars in LMC/SMC clusters (Aaronson and Mould 1985; Frogel et al. 1990) and with the cluster integrated infrared photometry (Persson et al. 1983). This will allow assessment of the fractional contribution of the infrared bright stars to the bolometric luminosity of each cluster. For the program clusters optical CCD observations have also been secured, the calibrated colourmagnitude diagram obtained, and an independent age determination for each cluster will soon be attempted (Buonanno et al. 1990).

\section{References}

Aaronson, M., Mould, J. (1985), Astrophys. J. 288, 551.

Buonanno, R., Corsi, C.E., Fusi Pecci, F., Greggio, L., Renzini, A., Sweigart, A.V. (1990), in prep.

Chiosi, C., Bertelli, G., Bressan, A. (1988), Astron. Astrophys. 196, 84.

Elson, R.A.W., Fall, S.M. (1985), Astrophys. J. 299, 211.

Frogel, J.A., Mould, R.J., Blanco, V.M. (1990), Astrophys. J. 352, 96.

Persson, S.E., Aaronson, M., Cohen, J.G., Frogel, J.A., Matthews, K. (1983), Astrophys. J. 266, 105.

Sweigart, A.V., Greggio, L., Renzini, A. (1990), Astrophys. J. in press. 